\title{
Numerical Investigation of Suction and Length of Suction Jet on Aerodynamic Characteristics of the NACA 0012 Airfoil
}

\author{
Kianoosh Yousefi, S. Reza Saleh, and Peyman Zahedi
}

\begin{abstract}
In this study, the effect of suction and the parameters affecting this process including suction amplitude, suction coefficient and the suction jet width in order to numeric flow control on an NACA 0012 airfoil was evaluated. Flow was fully turbulent with the Reynolds number of $5 \times 10^{5}$ and the turbulence employed model was the Menter's shear stress model. Suction on the airfoil was considered to be normal and uniform (perpendicular suction) and suction jet widths were 1.5, 2, 2.5 and 3 percent of the chord length. Based on previous studies, suction jet is located in optimal distance, 10 percent of the chord length from the leading edge. The range of suction jet entrance velocity was selected from 0.1 to 0.5 of freestream velocity. Results of this study demonstrated that the lift coefficient increased and drag coefficient decreased while suction amplitude rose. The maximum increase in lift to drag ratio was seen at suction amplitude of 0.5 . In addition, the lift to drag ratio elevated when suction jet width increased and reached to its maximum value at 2.5 percent of the chord length.
\end{abstract}

Index Terms-Suction, flow control, suction jet length, suction amplitude, lift and drag coefficient.

\section{INTRODUCTION}

The presence of boundary layer cause major problems in different fields of fluids mechanics. However, most of studies had focused on boundary layer effects on lift and drag forces, especially on wings. Developed methods for boundary layer management, lift coefficient increase and drag coefficient reduction are known as flow separation control or boundary layer control. The scope of flow separation control on an airfoil is to achieve more lift coefficient and less drag coefficient and consequently, airfoil higher performance by increasing the lift to drag ratio. Control methods of boundary layer are divided into two categories: passive flow separation control, requiring no auxiliary power and no control loop, and active flow separation control, requiring energy expenditure. Normal uniform suction and blowing which is among passive flow separation control, has been considered in recent years and most of studies have been concentrated on oscillatory suction or blowing near the leading edge. However, the effects of suction and blowing parameters variation that could provide a suitable research area, has not been considered appropriately.

Many studies have been conducted on flow separation

Manuscript received October 19, 2012; revised February 2, 2013.

Kianoosh Yousefi and Peyman Zahedi are with Mechanical Engineering, Mashhad Branch, Islamic Azad University, Mashhad, Iran (e-mail: k.yousefi@mshdiau.ac.ir and kianoosh_py@yahoo.com).

S. Reza Saleh is with School of Mechanical Engineering, Engineering Faculty, Mashhad Branch, Islamic Azad University, Mashhad, Iran (e-mail: s_r_saleh@yahoo.com). control. Prandtl was the first scientist who employed boundary layer suction to indicate its significant impacts on stream lines in 1904. He used suction on cylindrical surface to delay boundary layer separation. Boundary layer separation would be eliminated almost entirely by suction through a slot on the back of the cylinder [1]. First experiments on flow separation control on an airfoil were done in late 1930's to 1940. The effect of suction on boundary layer separation using slots on airfoil surface in wind tunnels was evaluated by NACA Langley memorial scientists. The first flight experiments in which seventeen suction slots were installed between 20 and 60 percent of the chord length was done. Employed airplane in this experiment was B-18 airplane [2]. Investigation on suction theoretical solution by Inverse boundary-Value problem was examined by Abzalilov et al. [3]. The efficiency of tangential unsteady suction and blowing in flow separation control on an airfoil TAU0015 was studied by Ravindran. He also evaluated the effects of Zero Net Mass Flux Oscillatory Jet (Synthetic Jet) on lift coefficient increase and flight conditions in his study were Mach 0.15, Reynolds number 1.2 million at the angle of attacks 22 and $24 \mathrm{deg}$. Result showed that Lift coefficient increased from 23 percent (angle of attack $22 \mathrm{deg}$ and suction coefficient is 0.0005 ) to 55 percent (angle of attack $24 \mathrm{deg}$ and same suction coefficient) [4].

Huang et al. studied on flow separation control on an NACA 0012 airfoil by using suction and blowing with angle of attack $18 \mathrm{deg}$ and Reynolds number of 5 Million in 2003 They proved that when jet location and angle of attack were combined, perpendicular suction at the leading edge, from 0.075 to 0.125 chord length, increased lift coefficient better than other suction situations. It has been also stated that tangential blowing at downstream locations, around 0.371 to 0.8 chord length, leads to the maximum increase in the lift coefficient value [5]. Resendiz investigated on the numerical simulation of flow separation control by oscillatory fluid injection and his result demonstrated that the use of synthetic jets on an NACA 0012 airfoil elevated the lift coefficient up to 93 percent [6]. The application of evolutionary algorithm in order to optimize the flow separation control has been studied by Beliganur \& Raymond in 2007. Results of their study showed that the use of two suction jets along with two blowing jets for an NACA 0012 airfoil was able to enhance the lift to drag ratio by 12 percent [7]. Flow separation control by synthetic jets on an NACA 0015 airfoil by using Large Eddy Simulation method was done in 2008 by You and Moin. Outcomes presented that lift coefficient increased 70 percent and drag coefficient decreased 18 percent while flow separation control parameters were changed [8]. Akcayoz \& 
Tuncer examined the optimization of synthetic jet parameters on an NACA 0015 airfoil in different angle of attack to maximize the lift to drag ratio and their results stated that optimum jet location moved toward leading edge and optimum jet angle went up while angle of attack increased [9]. Kim et al. used synthetic jets to flow separation control on an NACA 23012 airfoil. They focused on angle of attack, jet velocity and jet frequency for relatively high Reynolds numbers. This study showed, the maximum lift was obtained when the separation point coincided with the synthetic jet location and the non-dimensional frequency was one. Although the small vortex generated in the low frequency range beneficially affected the separation control and the lift enhancement, it caused the local flow structure to be easily destabilized by external disturbance or gust [10].

Piperas in 2010 studied on flow separation control on an NACA 4415 airfoil through different suction arrangements and increased the maximum lift coefficient value by 20 percent [11]. Genc et al. studied on the numerical effects of suction and blowing on the NACA 2415 airfoil at transition zone in 2011. Although separation bubbles were not entirely eliminated in suction and blowing simulation, they either reduced or moved into the downstream. For synchronic suction and blowing, separation bubbles were exterminated completely, lift coefficient increased and drag coefficient decreased. They also showed the best results were obtained with the single suction jet, intermediate results were obtained with the multi jets and the worst results were obtained with the blowing jets [12]. Yagiz et al. worked on drag optimization on Rae5243 airfoil in transonic conditions through suction. By optimum parameters selection they increased the lift coefficient, 3.17 percent, and decreased the total drag coefficient, 3.13 percent [13]. In addition, Yousefi et al. in 2012 reviewed the investigations on used methods in suction and blowing systems to increase or decrease drag and lift coefficient [14].

\section{GOVERNING EQUATIONS}

In this study the flow is assumed to be steady, incompressible and two-dimensional. So momentum and continuity equations become:

$$
\begin{gathered}
\frac{\partial u}{\partial x}+\frac{\partial v}{\partial y}=0 \\
\rho u \frac{\partial u}{\partial x}+\rho v \frac{\partial u}{\partial y}=-\frac{\partial P}{\partial x}+\frac{\partial}{\partial y}\left[\mu\left(\frac{\partial v}{\partial x}+\frac{\partial u}{\partial y}\right)\right] \\
\rho u \frac{\partial v}{\partial x}+\rho v \frac{\partial v}{\partial y}=-\frac{\partial P}{\partial y}+\frac{\partial}{\partial x}\left[\mu\left(\frac{\partial v}{\partial x}+\frac{\partial u}{\partial y}\right)\right]
\end{gathered}
$$

The Menter's shear stress transport turbulence model $(\mathrm{k}-\omega \mathrm{SST})$ was used to solve turbulence equations. This model which includes both $\mathrm{k}-\mathrm{w}$ and $\mathrm{k}-\varepsilon$ standard models, improved the calculations of boundary layer flows with separation and removed the sensitivity of $\mathrm{k}-\omega$ model in external flows. The transport equations in Menter's shear stress turbulence model are:

$$
\frac{\partial}{\partial t}(\rho k)+\frac{\partial}{\partial x_{i}}\left(\rho U_{i} k\right)=\widetilde{P}_{k}-\beta^{*} \rho k \omega+\frac{\partial}{\partial x_{i}}\left[\left(\mu+\sigma_{K} \mu_{t}\right) \frac{\partial k}{\partial x_{i}}\right]
$$

$$
\begin{aligned}
& \frac{\partial}{\partial t}(\rho \omega)+\frac{\partial}{\partial x_{i}}\left(\rho U_{i} \omega\right)=\alpha \rho S^{2}-\beta \rho \omega^{2}+ \\
& \frac{\partial}{\partial x_{i}}\left[\left(\mu+\sigma_{\omega} \mu_{t}\right) \frac{\partial \omega}{\partial x_{i}}\right]+2\left(1-F_{1}\right) \rho \sigma_{w 2} \frac{1}{\omega} \frac{\partial k}{\partial x_{i}} \frac{\partial \omega}{\partial x_{i}}
\end{aligned}
$$

In the equations (4) and (5), $\mathrm{F}_{1}$ is blending function, $\mathrm{S}$ is the invariant measure of the strain rate, $\beta^{*}$ is 0.09 and $\sigma_{\mathrm{w} 2}$ is 0.856. Blending function is equal to zero away from the surface $(\mathrm{k}-\varepsilon$ model), and switches over to one inside the boundary layer $\left(\mathrm{k}-\omega\right.$ model). A production limiter, $\widetilde{\mathrm{P}}_{\mathrm{k}}$, is used in the Menter's shear stress transport turbulence model to prevent the build-up of turbulence in stagnation regions. In addition, it is important to note that the all constants are computed by a blend from the corresponding constant of the $\mathrm{k}-\varepsilon$ and the $\mathrm{k}-\omega$ model via $\alpha, \sigma_{\mathrm{k}}, \sigma_{\omega}$ and etc [15 and 16].

$$
\begin{gathered}
F_{1}=\tanh \left\{\left\{\min \left[\max \left(\frac{\sqrt{k}}{\beta^{*} \omega y}, \frac{500 v}{y^{2} \omega}\right), \frac{4 \rho \sigma_{w 2} k}{C D_{k \omega} y^{2}}\right]\right\}\right\} \\
C D_{k \omega}=\max \left(2 \rho \sigma_{w 2} \frac{1}{\omega} \frac{\partial k}{\partial x_{i}} \frac{\partial \omega}{\partial x_{i}}, 10^{-10}\right) \\
P_{k}=\mu_{t} \frac{\partial U_{i}}{\partial x_{j}}\left(\frac{\partial U_{i}}{\partial x_{j}}+\frac{\partial U_{j}}{\partial x_{i}}\right) \\
\tilde{P}_{k}=\min \left(P_{k}, 10 \beta^{*} \rho k \omega\right)
\end{gathered}
$$

\section{PARAMETERS SELECTION}

In this study, the numerical code was used for simulation. Values for Reynolds number of flow and free stream velocity were $5 \times 10^{5}$ and $7.3037 \mathrm{~m} / \mathrm{s}$, respectively, and the used fluid was air. Geometry of NACA 0012 airfoil, suction jet location, suction angle and suction jet length has been shown in Fig. 1. The chord length of the airfoil is $1 \mathrm{~m}$ and suction slots located at 10 percent of the chord length from leading edge. Previous studies [5, 6, 8 and 9] shows that maximum lift to drag ratio obtains when suction jet is located at the given location. The suction jet lengths are 1.5, 2, 2.5 and 3 percent of the chord length and suction amplitude (the suction velocity to free stream velocity ratio) considered as $0.1,0.3$ and 0.5 in experiments. Thus, three investigate parameters are suction amplitude $(\mathrm{A})$, suction coefficient $\left(\mathrm{C}_{\mu}\right)$ and nondimensional suction jet length $(\mathrm{H})$. Examined angles of attack also are 12, 14, 16 and 18 degree. In our numerical investigation, the Suction amplitude and suction jet entrance velocity are set as:

$$
\begin{gathered}
A=\frac{u_{j}}{u_{\infty}} \\
u=A \cdot \cos (\theta+\beta) \\
v=A \cdot \sin (\theta+\beta)
\end{gathered}
$$

where $\beta$ is the angle between free stream velocity direction and the local jet surface and $\theta$ is also the angle between the local jet surface and jet entrance velocity direction. Note that negative $\theta$ represents suction condition and positive $\theta$ indicates blowing condition. Since perpendicular suction is 
investigated, $\theta$ is $-90 \mathrm{deg}$. Finally, suction coefficient equals:

$$
\begin{gathered}
C_{\mu}=\frac{\rho \cdot h \cdot v_{j}^{2}}{\rho \cdot C \cdot u_{\infty}^{2}}=\frac{h}{C} \times \frac{u_{j}^{2}}{u_{\infty}^{2}} \\
H=\frac{h}{C} \\
C_{\mu}=H \cdot A^{2}
\end{gathered}
$$

As it has been presented in equation (15), suction coefficient is related to two factors: suction amplitude (A) and suction jet length $(\mathrm{H})$. On the other hand variation of those values cause changes in suction coefficient value. Over 350 numerical simulations have been performed to cover all the cases.

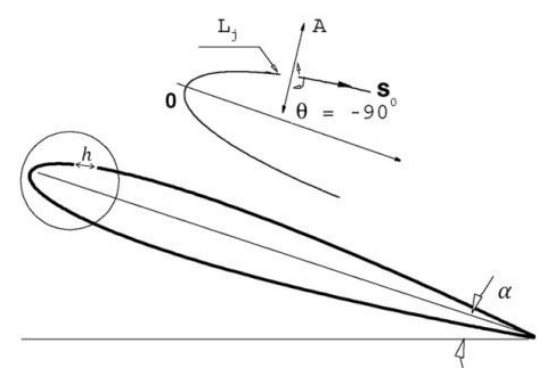

Fig. 1. Suction parameters on NACA 0012 Airfoil

\section{NUMERICAL SOLUTION METHOD}

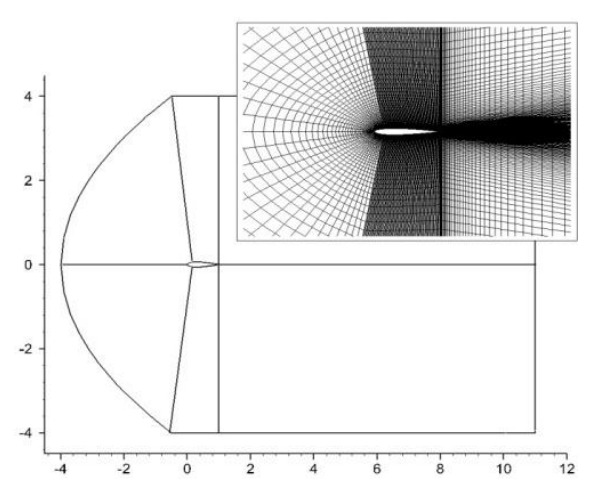

Fig. 2. C-type structured mesh with multizonal blocks

First and second order upwind method was employed to discretized the governing equations. First, equations are discrete by the use of first order upwind method, and the resulting system of equations is then solved using the SIMPLE method. Solution procedure is terminated when a convergence criteria of $\mathrm{O}(5)$ reduction in all dependent variable residuals is satisfied. Afterwards, second order upwind method was employed to discrete of equations and again, while SIMPLE method was employed to solve them. Convergence accuracy at this step is to the extent in which lift and drag coefficients fully converged, which happens usually at $\mathrm{O}(7)$. The key point here is that answers obtained from the first order upwind method was used as initial assumption for the second order upwind method. It is an attempt to consider the characteristics of laboratory wind tunnel, so the stream turbulence intensity is less than 0.1 percent. Airfoil computational area (C-type structured mesh) is considered as multizonal blocks in order to make structured mesh (Fig. 2).

The computational area grid extends from -4 chords upstream to 11 chords downstream and the upper and lower boundary extends 4 chords from the profile. In order to check the mesh independence of the calculated results, lift and drag coefficients have been studied at angles of attack 10,14 and 16 deg with different size grids. Table I presented lift and drag coefficients at angle of attack 16 deg and Fig. 3 and 4 showed meshes independent for different angles of attack. Consequently, the grid size giving the grid independent results is selected and the total number of cells is adopted as 41,000 nodes (Table I, Fig. 3 and 4).

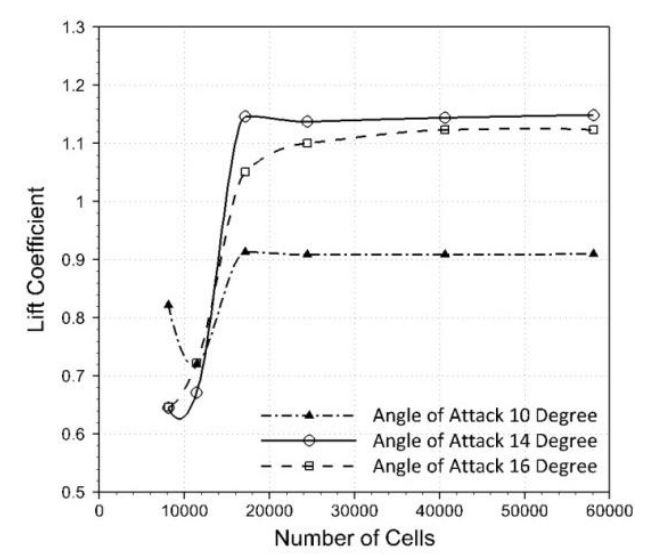

Fig. 3. Mesh independency for lift coefficient

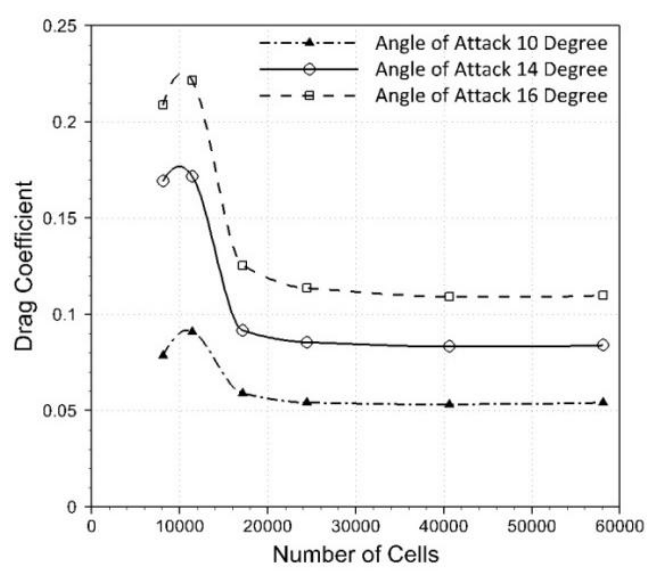

Fig. 4. Mesh independency for Drag coefficient

TABLE I: EVALUATION OF MESH INDEPENDENCE AT ANGLE OF ATTACK 16 DEG

\begin{tabular}{ccc}
\hline \multicolumn{3}{c}{ ATTACK 16 DEG } \\
\hline number of meshes & lift coefficient & drag coefficient \\
\hline 8096 & 0.64594 & 0.20889 \\
17160 & 1.05134 & 0.12544 \\
24480 & 1.09073 & 0.11567 \\
40640 & 1.12352 & 0.10938 \\
58080 & 1.12319 & 0.11187 \\
\hline \hline
\end{tabular}



Fig. 5. Lift coefficient convergence for angle of attacks $12^{\circ}$ and $18^{\circ}$ 
As demonstrated in Fig. 5 and 6, the solutions in all cases, continued until lift and drag coefficient fully converged. Then, the results were compared with the results of numerical solution of Huang et al. [5] and experimental values of Critzos et al. [17]. Huang et al. investigated on flow separation control using suction and blowing on NACA 0012 airfoil where the angle of attack and Reynolds number were $18 \mathrm{deg}$ and $5 \times 10^{5}$, respectively. Parameters like jet location, suction and blowing amplitude and angle of attack were also examined by numerical method. In order to model the suction, a jet with 2.5 percent of the chord length as width was placed on the upper surface of airfoil. The GHOST code, based on finite volume, was used in this study. Critzos et al. examined aerodynamic characteristics of a NACA 0012 airfoil in laboratory experiments where Reynolds numbers were $0.5 \times 10^{5}$ and $1.8 \times 10^{6}$ and the angles of attack changed from 0 to $180 \mathrm{deg}$. The results of these three solutions are compared in Fig. 7. As it is seen, performed numerical solution results are very close to the given results of Huang et al. The highest recorded error was 8 percent, at 14 deg angle of attack. Also stall angle in both method were angle of attack $14 \mathrm{deg}$. However, the results of laboratory measurements indicated that NACA 0012 airfoil stall occurs at 12 deg angle of attack. It also important that turbulence model selection has a significant influence on stall angle changes. So, the selection of $\mathrm{K}-\varepsilon$ two-equation turbulence model at the same condition changes the stall angle to $16 \mathrm{deg}$. Menter's shear stress transport turbulence model always gives better results than $\mathrm{K}-\varepsilon$ two-equation model. In the $\mathrm{K}-\varepsilon$ model, the maximum error at the angle of attack $14 \mathrm{deg}$ for lift coefficient and drag coefficient were 17 percent and 25 percent, respectively. In addition, the results of performed studies showed that although Menter's shear stress transport turbulence model is more suitable model for lower Reynolds number, with larger Reynolds number $\mathrm{K}-\varepsilon$ model gives more reliable results.

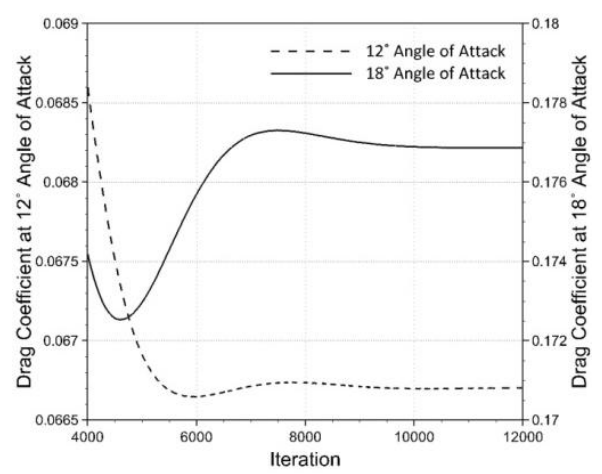

Fig. 6. Drag coefficient convergence for angle of attacks $12^{\circ}$ and $18^{\circ}$

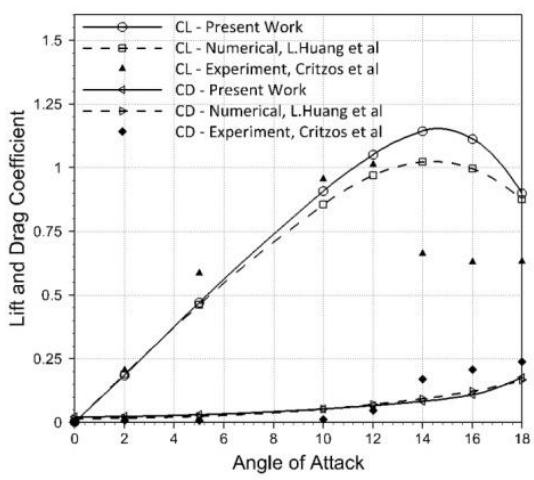

Fig. 7. Comparison between present work with numerical [5] and experimental [17] results

\section{RESULTS AND DISCUSSION}

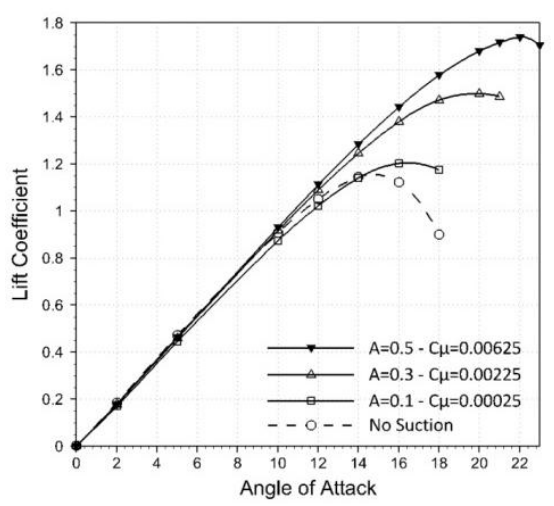

Fig. 8. The effects of suction amplitude and suction coefficient on lift coefficient and compare with no suction condition

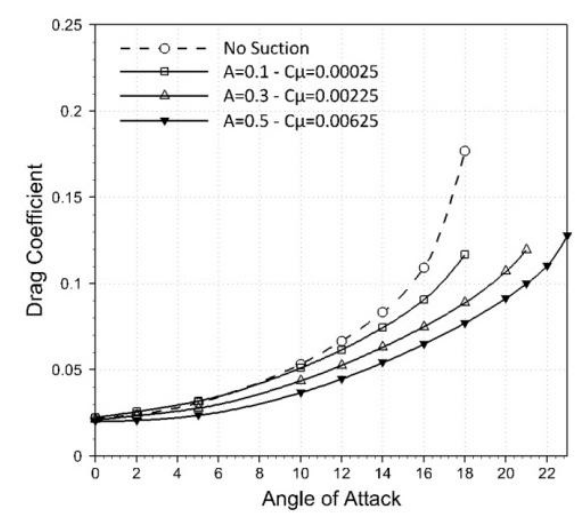

Fig. 9. The effects of suction amplitude and suction coefficient on drag coefficient and compare with no suction condition

First, the effects of suction amplitude and suction coefficient on lift and drag coefficients were evaluated (Fig. 8 and 9). The suction jet length was 2.5 percent of the chord length in order to obtain accurate diagrams. Assumed suction amplitude was $0.1,0.3$ and 0.5 and suction coefficient values in this study were $0.00025,0.00225$ and 0.00625 . Lift coefficient increased and drag coefficient decreased while suction coefficient increased. However the amount of lift coefficient increase at the angles of attack lower than $10 \mathrm{deg}$, was insignificant. For instance, lift drag increase value was just 2.4 percent when suction coefficient reached to 0.00625 . It is significant to remember is that the effect of drag coefficient reduction is more significant and at the given suction coefficient $(0.00625)$, when the angle of attack was $10 \mathrm{deg}$, showed 28 percent drop. The maximum lift to drag ratio increase was seen with the value of suction coefficient of 0.00625 and this amount was 75 percent at 18 deg angle of attack. At the given angle of attack, lift coefficient raised 43 percent and drag coefficient declined 56 percent. Incremental changes of lift coefficient went down with increasing suction coefficient. For example, when suction coefficient changed from 0.00025 to 0.00225 at the $18 \mathrm{deg}$ angle of attack, lift coefficient raised 20 percent approximately and the amount of drag coefficient reduction was 24 percent; however, while suction coefficient was increasing from 0.00225 to 0.00625 , lift coefficient increased just 5 percent and the values of drag coefficient dropped 12 percent. It is also significant that increasing suction coefficient leads to stall angle boost. As it has been shown, when there was no suction, stall happened at 14 deg angle of attack, but stall angle were 16, 20 and 22 deg while suction coefficients moved from 0.00025 to 0.00225 
and then 0.00625 respectively. Therefore, due to the use of perpendicular suction, not only the lift to drag ratio increased, but also stall angle was delayed and changed from $14 \mathrm{deg}$ (No suction condition) to $22 \mathrm{deg}$. Fig. 10 shows stream lines around the airfoil with the angle of attack $18 \mathrm{deg}$. Suction coefficient increase caused the reduction of vortex formation behind the airfoil and they disappeared eventually.

Fig. 11, Fig. 12 and Fig. 13 show the lift coefficient changes, drag coefficient variations and the lift to drag ratio with suction jet length at suction amplitude of 0.3 and different angles of attack. As it is noticeable, although drag and lift coefficients were almost constant at the low angles of attack, even when the suction jet length changed, their values showed significant changes at increased angles of attack. Lift coefficient increased and drag coefficient decreased because of suction jet length increase. This increase continued until the suction jet length reached to 2.5 percent of the chord length and after that it showed insignificant reduction between 2.5 and 3 percent of the chord length. By increasing the suction jet length from 1.5 to 2.5 percent of the chord length, lift coefficient increased 6.3 percent and drag coefficient reduced 15 percent. However, suction jet length increase from 2.5 to 3 percent of the chord length lead to only 0.75 percent lift coefficient increase and drag coefficient remained almost constant. The lift to drag ratio had almost similar situation. In this case and at the angle of attack $18 \mathrm{deg}$, although increasing suction jet length from 1.5 to 2.5 percent of the chord length caused 24 percent increase in the value of the lift to drag ratio, when the length of suction jet elevated from 2.5 to 3 percent of the chord length, the lift to drag ratio went down only 2 percent. Thus, optimum suction jet length was 2.5 to 3 percent of the chord length. Danenberg \& Weiberg [18] who have conducted experimental investigations on symmetrical airfoil with a thickness of 10.51 percent of the chord length (NACA airfoil 0010.51) and 2.3 and 6.3 of the chord length as the length of suction jet, demonstrated that the optimum suction jet length would be 2.3 percent of the chord length and the maximum lift of the symmetrical 10.51 percent thick wing was increased from a lift coefficient approximately 1.3 to approximately 1.8 by means of area suction over the first three percent of chord. The result of that study confirmed obtained data in present investigation.

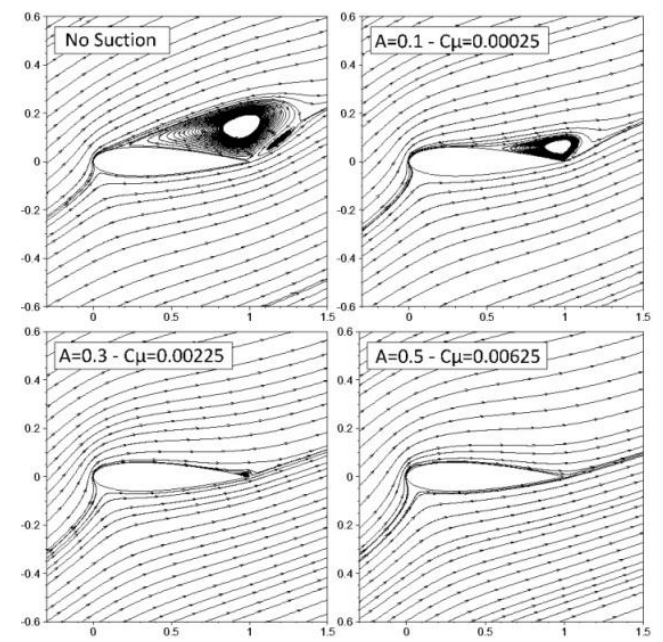

Fig. 10. Stream lines around the airfoil at the angle of attack $18 \mathrm{deg}$ and different suction coefficient

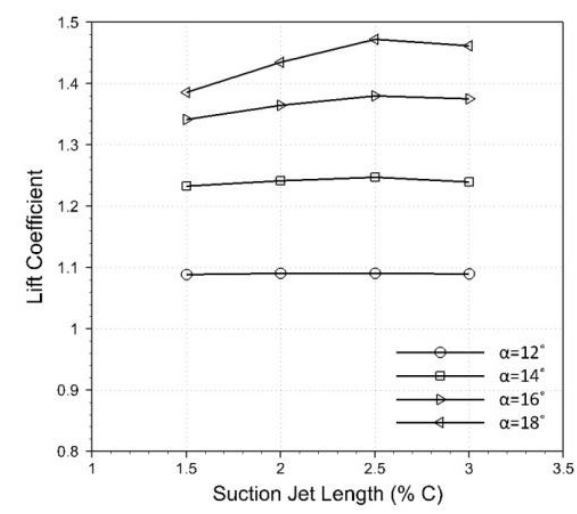

Fig. 11. Lift coefficient changes in different suction jet length with suction amplitude of 0.3 and angle of attack $12^{\circ}, 14^{\circ}, 16^{\circ}$ and $18^{\circ}$

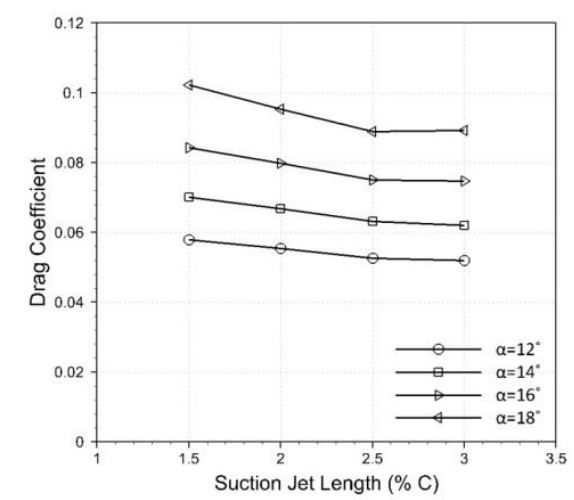

Fig. 12. Drag coefficient changes in different suction jet length with suction amplitude of 0.3 and angle of attack $12^{\circ}, 14^{\circ}, 16^{\circ}$ and $18^{\circ}$

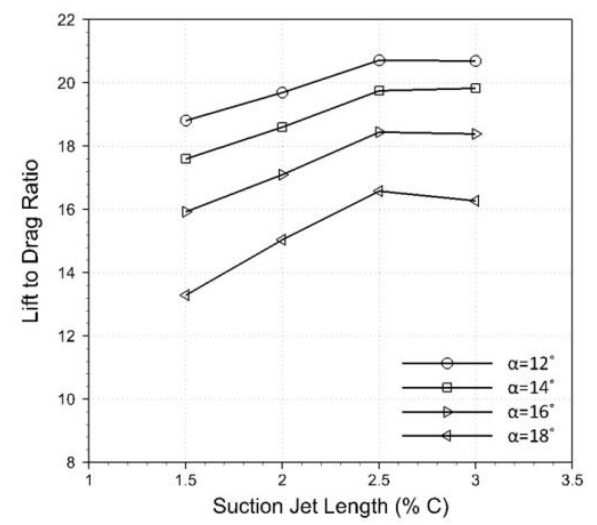

Fig. 13. Lift to drag ratio changes in different suction jet length with suction amplitude of 0.3 and angle of attack $12^{\circ}, 14^{\circ}, 16^{\circ}$ and $18^{\circ}$

Figures 14, 15 and 16 present the relations between lift coefficient changes, drag coefficient variations and the lift to drag ratio with suction jet length at suction amplitude of 0.5 and different angles of attack. In this situation also increase in the lift coefficient continued until the suction jet length reached to 2.5 percent of the chord length and then it declined slightly between 2.5 and 3 percent of the chord length. However, increasing suction amplitude value from 0.3 to 0.5 caused more reduction of drag coefficient and increase of lift coefficient. For instance, at the angle of attack $18 \mathrm{deg}$, when suction jet length changed from 2.5 to 3 percent of the chord length, lift coefficient declined 1.4 percent, drag coefficient rose 1 percent and the lift to drag ratio decreased 2.5 percent. In this case also drag and lift coefficients fluctuations, as well as the lift to drag ratio changes were insignificant at the lower angles of attack. Fig. 17 describes the effects of suction jet length on vortex behind the airfoil at the angle of attack 16 
deg and as it has been cleat, when the suction jet lengths were 2.5 and 3 percent of the chord length, eddies were omitted entirely and separation was not happened.

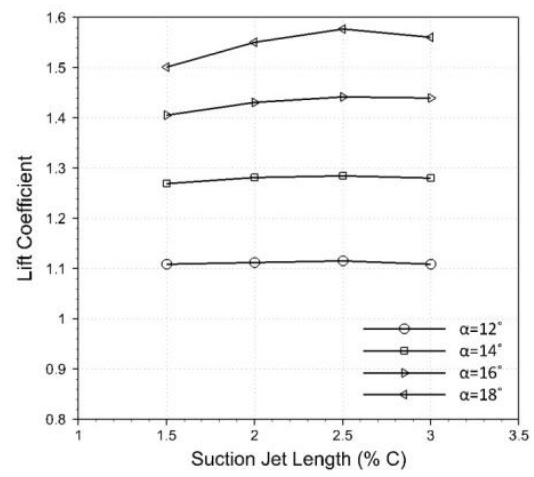

Fig. 14. Lift coefficient changes in different suction jet length with suction amplitude of 0.5 and angle of attack $12^{\circ}, 14^{\circ}, 16^{\circ}$ and $18^{\circ}$



Fig. 15. Drag coefficient changes in different suction jet length with suction amplitude of 0.5 and angle of attack $12^{\circ}, 14^{\circ}, 16^{\circ}$ and $18^{\circ}$

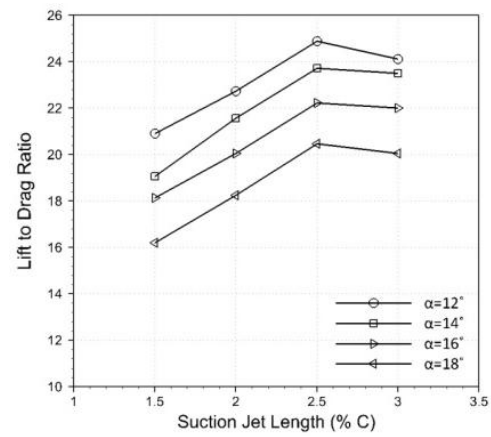

Fig. 16. Lift to drag ratio changes in different suction jet length with suction amplitude of 0.5 and angle of attack $12^{\circ}, 14^{\circ}, 16^{\circ}$ and $18^{\circ}$

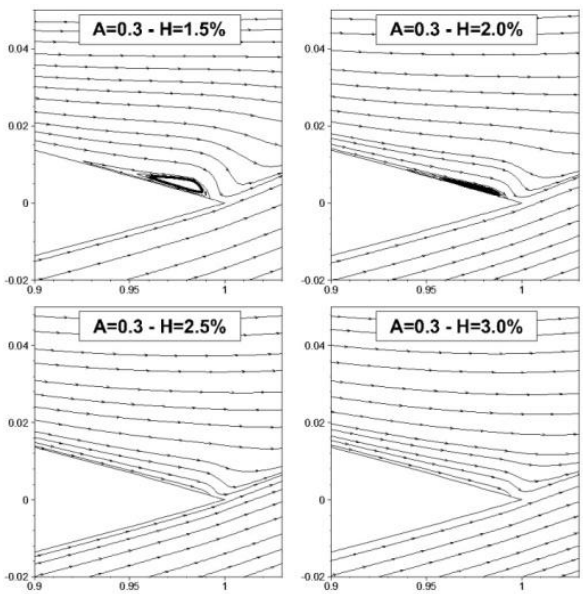

Fig. 17. Effect of suction jet length on eddies behind the airfoil at $16 \mathrm{deg}$ angle of attack

\section{CONCLUSION}

In this work, the effects of suction on an NACA 0012 airfoil for flow separation control were presented and analyzed. Thus, numerical simulation was employed to measure the effects of suction amplitude and suction jet length fluctuations. Results showed that the lift to drag ratio increased when suction amplitude enhanced and the separation point transferred to downstream. The maximum lift to drag ratio value obtained when suction amplitude and suction coefficient reached to 0.5 and 0.00625 , respectively. At the angle of attack $18 \mathrm{deg}$ and same suction amplitude and suction coefficient, vortex behind the airfoil was eliminated entirely. Another significant point, as it has been described is that the flow separation control using suction had no significant influence on aerodynamic characteristics at low angles of attack. In addition, the use of suction on airfoil could raise the airfoil stall angle. In this investigation, stall angle changed from 14 to $22 \mathrm{deg}$ while suction coefficient reached to 0.00625 .

Another outstanding analyzed factor was the effect of suction jet length variations on airfoil surface. Obtained results presented that the lift to drag ratio went up by increasing suction jet length and separation point transferred to downstream. Lift coefficient increase continued until the length of suction jet reached to 2.5 percent of the chord length and then it declined slightly between 2.5 and 3 percent of the chord length. Finally, the airfoil lift to drag ratio boost 75 percent and stall angle reached to $22 \mathrm{deg}$ at suction amplitude of 0.5 , the angle of attack $18 \mathrm{deg}$, and 2.5 percent of the chord length as suction jet length. Also, the lift to drag ratio at angle of attack $18 \mathrm{deg}$ and suction amplitude of 0.5 increased 26 percent when suction jet length changed from 1.5 to 2.5 percent of the chord length.

\section{REFERENCES}

[1] M. Gad-El-Hak, Control Flow: Passive, Active and Reactive Flow Management, Cambridge University Press, United Kingdom, 2000

[2] A. L. Braslow, "A history of suction type laminar flow control with emphasis on flight research," NASA History Division, Monograph in Aerospace History, no. 13, 1999.

[3] D. F. Abzalilov, L. A. Aksentev and N. B. IL'Inskii, "The inverse Boundary-Value problem for an airfoil with a suction slot," Journal of Applied Mathematics and Mechanics, vol. 61, pp. 75-82, 1997.

[4] S. S. Ravindran, "Active control of flow separation over an airfoil," Report of Langley Research Center, 1999.

[5] L. Huang, P. G. Huang, and R. P. LeBeau, "Numerical study of blowing and suction control mechanism on NACA0012 airfoil," Journal of Aircraft, vol. 41, 2004.

[6] C. R. Rosas, "Numerical simulation of flow separation Control by oscillatort fluid injection," Doctor of Philosophy Thesis, A\&M University, Texas, 2005.

[7] N. K. Beliganur and P. Raymond, "Application of evolutionary algorithms to flow control optimization," Report of University of Kentuchky, 2007.

[8] D. You and P. Moin, "Active Control of flow Separation over an airfoil using synthetic jets," Journal of Fluids and Structures, vol. 24, pp. 1349-1357, 2008.

[9] E. Akcayoz and I. H. Tuncer, "Numerical investigation of flow control over an airfoil using synthetic jets and its optimization,' Presented at International Aerospace Conference, Turkey, 2009.

[10] S. H. Kim and C. Kim, "Separation control on NACA23012 using synthetic jet," Aerospace Science and Technology, vol. 13, pp. 172-182, 2009.

[11] A. T. Piperas, "Investigation of boundary layer suction on a wind turbine airfoil using CFD," Master Thesis, Technical University of Denmark, Denmark, 2010.

[12] M. S. Genc, U. Keynak, and H. Yapici, "Performance of Transition Model for Predicting Low Re Aerofoil Flows Without/With Single 
and Simultaneous Blowing and Suction," European Journal of Mechanics B/Fluids, vol. 30, pp. 218-235, 2011.

[13] B Yagiz, O. Kandil, and Y. V. Pehlivanoglu, "Drag minimization using active and passive flow control techniques," Aerospace Science and Technology, vol. 17, pp. 21-31, 2011.

[14] K. Yousefi, S. R. Saleh, and P. Zahedi, "Investigation for Increase or Decrease The Lift and Drag Coefficient on The Airfoil with Suction and Blowing," International Conference on Mechanical Engineering and Advanced Technology, Iran, 2012.

[15] F. R. Menter, M. Kuntz, and R. Langtry, "Ten Years of Industrial Experience with the SST Turbulence Model," 4th International Symposium on Turbulence, Heat and Mass Transfer, Turkey, 2003.

[16] L. K. Voigt, J. N. Sorensen, J. M. Pedersen, and M. Crons, "Review of Four Turbulence Models Using Topology," presented at 8th International IBPSA Conference, Netherlands, 2003.

[17] C. C. Critzos, H. H. Heyson, and W. Boswinkle, "Aerodynamics Characteristics of NACA0012 Airfoil Section at Angle of Attacks from $0^{\circ}$ to $180^{\circ}$, " Langley Aeronautical Laboratory, Washington, NACA Technical Note 3361, 1955.

[18] R. E. Dannenberg and J. A. Weiberg, "Section Characteristics of a 10.5 Percent Thick Airfoil with Area Suction as Affected by Chordwise Distribution of Permeability," NASA Technical Note 2847, Washington, 1952.

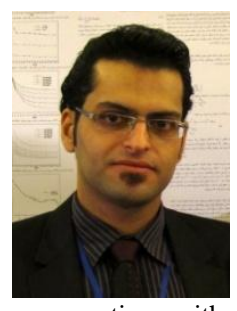

Kianoosh Yousefi was born in 1986, Gorgan, Iran He received associate degree in industrial design from Azad University of AliAbad, Gorgan, Iran in 2007 and B.Sc. degree in mechanical engineering the field of solids design from Azad university of Mashhad, Mashhad, Iran in 2009 with high distinction. Currently he is M.Sc. student (final semester) in Azad University of Mashhad and studied mechanical engineering the field of energy conversation with great distinction. His research interests include Aerodynamics, Experimental Aerodynamics, Boundary Layer Control, Boundary Layer Theory, Fluid Mechanics, Turbulent Flows and Modeling. His primary research areas of interest are aerodynamics characteristics of the wings, flows about the wings and boundary layer control in experimental.

Mr. Yousefi is a member of ASME, ISME, IAS and Iranian combustion institute (ICI). Some of the most important paper titles that presented in ICMEAT 2012 conference which held in Isfahan, Iran are "Investigation for increase or decrease the lift and drag coefficient on the airfoil with suction and blowing", "Numerical investigation on the flame speed of $\mathrm{CH}_{4} /$ Air diluted with $\mathrm{CO}_{2}$ and vapor", "Incompressible fluid flow on a circular cylinder with heat transfer by finite element method" and "Incompressible fluid flow on four elliptic cylinder by finite element method". 